\title{
Realizing Optical Persistent Spin Helix and Stern-Gerlach Deflection in an Anisotropic Liquid Crystal Microcavity
}

\author{
Mateusz Królø, ${ }^{1, *}$ Katarzyna Rechcińska $\odot,{ }^{1, *}$ Helgi Sigurdsson $\odot,{ }^{2,3,4}$ Przemysław Oliwa $\odot,{ }^{1}$ Rafał Mazur $\odot{ }^{5}$ \\ Przemysław Morawiak $\odot,{ }^{5}$ Wiktor Piecek $\odot,{ }^{5}$ Przemysław Kula $\odot,{ }^{6}$ Pavlos G. Lagoudakis $\odot,{ }^{2,3}$ Michał Matuszewski®, ${ }^{7}$ \\ Witold Bardyszewski®, ${ }^{8}$ Barbara Piętka $\odot,{ }^{1}$ and Jacek Szczytko๑ ${ }^{1, \dagger}$ \\ ${ }^{1}$ Institute of Experimental Physics, Faculty of Physics, University of Warsaw, Pasteura 5, PL-02-093 Warsaw, Poland \\ ${ }^{2}$ Skolkovo Institute of Science and Technology, Bolshoy Boulevard 30, building 1, Moscow 121205, Russia \\ ${ }^{3}$ Department of Physics and Astronomy, University of Southampton, Southampton SO17 1BJ, United Kingdom \\ ${ }^{4}$ Science Institute, University of Iceland, Dunhagi 3, IS-107 Reykjavik, Iceland \\ ${ }^{5}$ Institute of Applied Physics, Military University of Technology, Kaliskiego 2, PL-00-908 Warsaw, Poland \\ ${ }^{6}$ Institute of Chemistry, Military University of Technology, Kaliskiego 2, PL-00-908 Warsaw, Poland \\ ${ }^{7}$ Institute of Physics, Polish Academy of Sciences, al. Lotników 32/46, PL-02-668 Warsaw, Poland \\ ${ }^{8}$ Institute of Theoretical Physics, Faculty of Physics, University of Warsaw, Pasteura 5, PL-02-093 Warsaw, Poland
}

(Received 26 May 2021; accepted 24 August 2021; published 1 November 2021)

\begin{abstract}
Spin-orbit interactions which couple the spin of a particle with its momentum degrees of freedom lie at the center of spintronic applications. Of special interest in semiconductor physics are Rashba and Dresselhaus spin-orbit coupling. When equal in strength, the Rashba and Dresselhaus fields result in SU(2) spin rotation symmetry and emergence of the persistent spin helix only investigated for charge carriers in semiconductor quantum wells. Recently, a synthetic Rashba-Dresselhaus Hamiltonian was shown to describe cavity photons confined in a microcavity filled with optically anisotropic liquid crystal. In this Letter, we present a purely optical realization of two types of spin patterns corresponding to the persistent spin helix and the SternGerlach experiment in such a cavity. We show how the symmetry of the Hamiltonian results in spatial oscillations of the spin orientation of photons traveling in the plane of the cavity.
\end{abstract}

DOI: 10.1103/PhysRevLett.127.190401

The stability of a number of interesting phenomena in physical systems can be explained as a consequence of certain underlying symmetries that are robust to perturbations. Out of many examples in solid-state physics, one that in recent years has attracted a great deal of attention is the persistent spin helix protected by an unusual SU(2) symmetry [1]. It emerges in two-dimensional semiconductors exhibiting Rashba and Dresselhaus spin-orbit coupling (SOC) of equal magnitudes. The persistent spin helix (PSH) is characterized by a spatially periodic spin texture of the SOC particles which become robust against spindependent scattering, with spin relaxation suppressed. So far, this effect has been experimentally demonstrated in many implementations [2-9]. It was first observed through transient spin-grating spectroscopy [2], then directly mapped in an optical Kerr rotation experiment [3].

In the context of SOC of light [10], a rapidly growing field of research, there has been so far no implementation of a

Published by the American Physical Society under the terms of the Creative Commons Attribution 4.0 International license. Further distribution of this work must maintain attribution to the author(s) and the published article's title, journal citation, and DOI. photonic PSH to protect the polarization state of light. Today, SOC of light has led to remarkable results aiming to study well-known concepts in solid-state electronic systems but in a new optical context. This has contributed to the development of exciting areas of study such as topological photonics [11], nontrivial and singular polarization textures [12,13], optical spin-based metamaterials [14], valleytronics [15,16], and synthesizing artificial gauge fields in photonic lattices [17].

In this Letter, we realize both a photonic PSH as well as an optical Stern-Gerlach experiment using a liquid crystalfilled multimode cavity. By tuning two modes of opposite parity and polarization into resonance in this highly anisotropic cavity, their mixing becomes described by an effective equal Rasha-Dresselhaus SOC, resulting in a dispersion with strong valley polarization [18]. Our experimental observations and analytical calculations demonstrate that the strong polarization-valley coupling in this simple system directly results in the appearance of longrange polarization [or (pseudo)spin] textures of the in plane traveling photons with potential application for valleyoptronic devices [19-21] alongside gapped Dirac materials. In Rashba-Dresselhaus SOC conditions the polarization of light depends only on the distance traveled along one direction and not on the initial momentum; thus, parallel stripes of linear polarization are formed. 

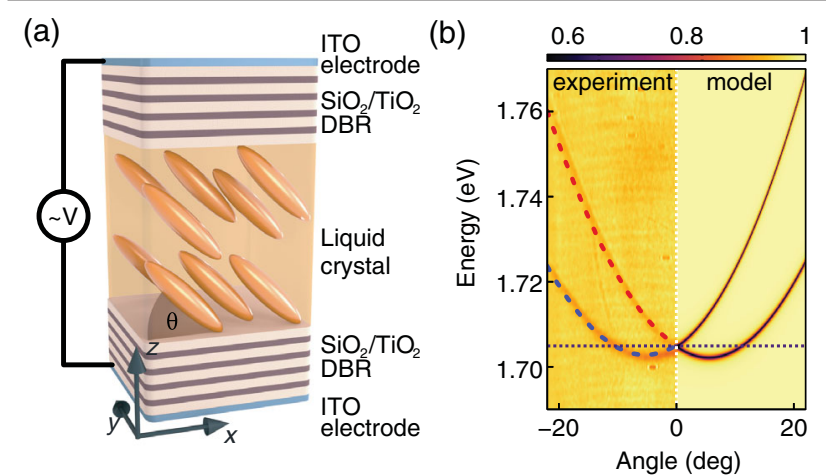

(c)

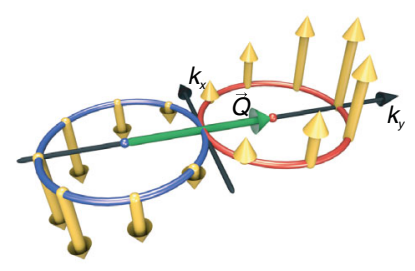

(d)

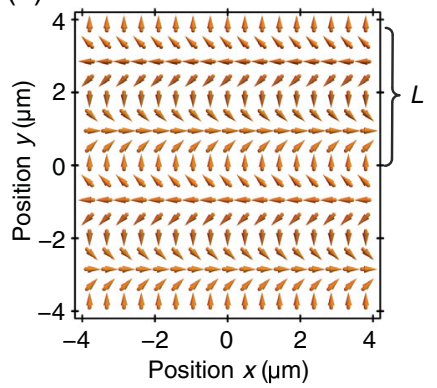

FIG. 1. The effect of liquid crystal birefringence tuning. (a) Scheme of the cavity filled with nematic liquid crystal. With external voltage applied to LCMC, the mean orientation of LC molecules tilts in the $x-z$ plane. (b) Angle-resolved reflectance spectra in $y$ direction for Rashba-Dresselhaus resonance in LCMC: experiment and Berreman matrix simulation. Blue and red dashed lines mark fitted Rashba-Dresselhaus dispersion relation for spin-up and spin-down photons, respectively. Dashed horizontal line marks energy of the laser used for resonance transmission measurements. (c) Constant energy cross section through Rashba-Dresselhaus dispersion relation which consists of two circularly polarized circles off centered by $\vec{Q}$ (green arrow). Yellow arrows denote the effective SOC magnetic field given by the last term in Eq. (1). (d) Schematic illustration of persistent spin helix spin texture with period $L=3.8 \mu \mathrm{m}$.

We use a microcavity filled with a liquid crystalline medium shown schematically in Fig. 1(a). The cavity is based on two $\mathrm{SiO}_{2} / \mathrm{TiO}_{2}$ distributed Bragg reflectors (DBRs), with maximum reflectance at $1.65 \mathrm{eV}(750 \mathrm{~nm})$. Approximately $3.5 \mu \mathrm{m}$ gap between the DBRs is filled with a nematic liquid crystal of high birefringence $(\Delta n=0.41$ $[22,23])$, which acts as an optically uniaxial medium inside a multimode cavity. By tuning an external voltage applied to transparent ITO electrodes, we can control the anisotropy direction in the $x-z$ plane [Fig. 1(a)], which changes the effective refractive index and thus the cavity mode energy for light polarized in the $x$ direction, whereas modes of perpendicular polarization are unaffected [24]. If the refractive indices for perpendicular linear polarizations are different, then degeneration of two modes with different numbers is possible. When two modes of opposite parities are degenerate, they couple via the Rashba-Dresselhaus SOC term, and the dispersion can be described by an effective Hamiltonian written in the photon circular polarization basis (i.e., spin-up and spin-down states) [18]:

$$
\hat{H}=\frac{\hbar^{2} \vec{k}^{2}}{2 m}-2 \alpha \hat{\sigma}_{z} k_{y},
$$

which is the same as considered by Bernevig et al. in [1]. Here, $\vec{k}=\left(k_{x}, k_{y}\right)$ is the cavity in plane momentum, and $\hat{\sigma}_{z}$ is the third Pauli matrix. The above Hamiltonian can be regarded as a consequence of emergent chirality, or optical activity, in the structure which-alongside other SOC mechanisms-leads to nontrivial band geometry [25]. It is worth mentioning that the form of the term linear in $k$ is induced by the symmetry of the problem, and in this sense, it is robust (see the Supplemental Material in [18]).

The Rashba-Dresselhaus SOC dispersion was achieved when $2.12 \mathrm{~V}$ AC voltage was applied to the sample. Dispersion relation of photons confined in the cavity can be mapped directly through angle-resolved reflectance spectra, as shown in Fig. 1(b). The dispersion for wave vectors along the $y$ direction shows two off-centered spinpolarized parabolas (or valleys), where the constant energy cross section consists of two spin circles off-centered by momentum $\vec{Q}=4 m \alpha / \hbar^{2} \hat{y}$ [Fig. 1(c)], with blue and red colors denoting spin-down and spin-up states, and yellow arrows indicating the effective momentum-dependent outof-plane magnetic field. Fitting Eq. (1) to the dispersion gives a Rashba parameter $\alpha=2.8 \times 10^{-3} \mathrm{eV} \mu \mathrm{m}$ and effective mass $m=1.1 \times 10^{-5} m_{\mathrm{e}}$ where $m_{e}$ is the free electron rest mass. The angle-resolved reflectance spectra was also simulated numerically using the Berreman method [26,27]. The results of such simulations for a liquid crystal (LC) layer width of $3.1 \mu \mathrm{m}$ with molecules rotated by $\theta=53.3^{\circ}$ are compared with the experiment in Fig. 1(b).

Interestingly, one can consider the equal RashbaDresselhaus SOC system as a spin realization of Young's double slit experiment in a reciprocal space. The role of the two slits discriminating the position of an incident scalar plane wave in the real space-resulting in the well-known double-slit spatial interference pattern in the far field - is instead played by the two cavity dispersion spin valleys in reciprocal space discriminating the momenta of light through an optical mode [Fig. 1(c)]. In the doubleslit experiment a plane wave passing through two slits (separated by distance $d$ ) produces spherical waves that interfere, giving an image of the intensity oscillating in space with a period proportional to $1 / d$ [Fig. 2(a)]. Analogously, for a homogeneous occupation of the two isoenergy spin circles in the reciprocal space, separated by the vector $\vec{Q}$, we would obtain in real space a polarization interference image producing the persistent spin helix with a period proportional to $L=2 \pi / Q$ where $Q=|\vec{Q}|$. In other words, in the Young's double slit experiment, one 
obtains in the far field the Fourier transform of the two slits, which is a periodic interference pattern. Here, we obtain in the near field the Fourier transform of the two polarized circles in momentum space, which is a linear polarization interference pattern. Based on the fitted dispersion in Fig. 1(b) this leads to a helix period of $L=3.8 \mu \mathrm{m}$ [Fig. 1(d)].

This change of the polarization with propagation of photons in the plane of the cavity is observed experimentally in our system. An incident linearly antidiagonally polarized laser beam is tightly focused with a microscope objective $(100 \times$ magnification, 0.55 numerical aperture) on the sample, providing homogeneous occupation of photons on both spin circles in reciprocal space. The laser energy is set resonant with the cavity modes at normal incidence as marked by a dashed horizontal line in Fig. 1(b). Transmitted light is collected by another microscope objective, and polarization is resolved and imaged on a camera. This allows us to map out the spatial distributions of the $S_{1}=\left(I_{X}-\right.$ $\left.I_{Y}\right) /\left(I_{X}+I_{Y}\right)$ and $S_{2}=\left(I_{d}-I_{a}\right) /\left(I_{d}+I_{a}\right)$ and $S_{3}=$ $\left(I_{\sigma^{+}}-I_{\sigma^{-}}\right) /\left(I_{\sigma^{+}}+I_{\sigma^{-}}\right)$Stokes parameters, corresponding to intensities of horizontal $\left(I_{X}\right)$, vertical $\left(I_{Y}\right)$, diagonal $\left(I_{d}\right)$, antidiagonal $\left(I_{a}\right)$, right-hand circular $\left(I_{\sigma^{+}}\right)$, and lefthand circular $\left(I_{\sigma^{-}}\right)$polarized light.

The measured $S_{1}$ and $S_{2}$ parameters are plotted in Figs. 2(c) and 2(f), which clearly show periodic oscillations with a $\pi / 2$ phase shift between the two Stokes parameters. The spatial period of the oscillations is estimated as $L=4.7 \mu \mathrm{m}$. The phase of the PSH depends on the polarization of incident light, as described in the Supplemental Material [28].

As mentioned above, this result can be understood as a consequence of an interference process between spins in different momentum valleys (i.e., valley polarization). It is worth mentioning another type of photonic SOC inherent to planar microcavities - known as the optical spin Hall effect-which comes from different transmission and reflection properties of TE and TM polarized modes of the same mode number $[12,24]$. It also leads to spatially beating polarization patterns due to the different effective masses of the linearly polarized modes but is inherently nonchiral, and thus very different in origin from Eq. (1) whose eigenmodes are circularly polarized. Assuming that the cavity extends infinitely in the $x-y$ plane with the two almost perfect mirror planes separated by distance $L$, we can represent the modal electric fields inside the cavity, corresponding to the eigenvalues $\varepsilon^{ \pm}(\vec{k})=\hbar^{2} / 2 m(\vec{k} \mp \vec{Q} / 2)^{2}-\left(2 m \alpha^{2} / \hbar^{2}\right)$ of the Hamiltonian [Eq. (1)] by the plane waves:

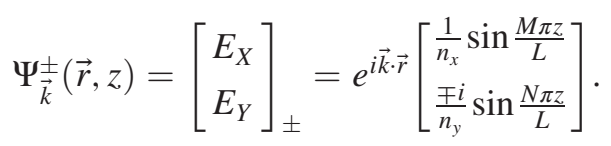
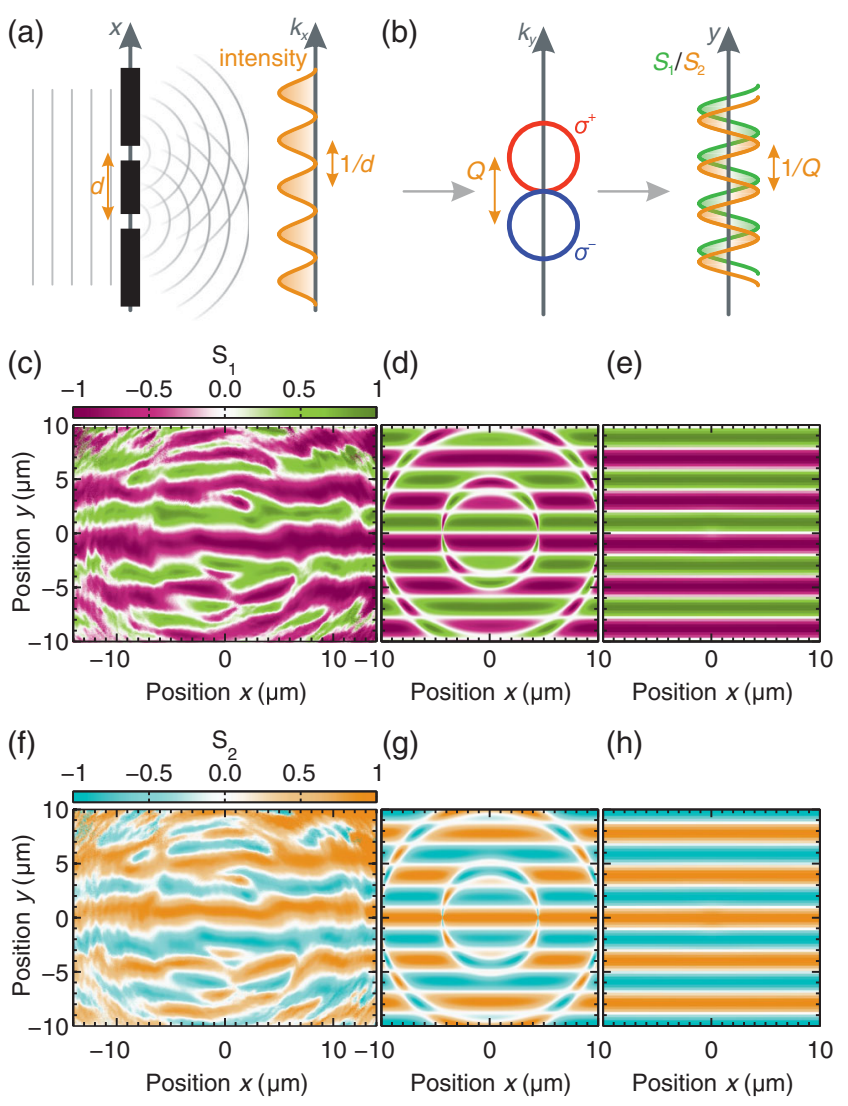

FIG. 2. All-optical persistent spin helix state. (a) Schematic of Young's double slit experiment. (b) Schematic of the reciprocal spin-valley interference experiment. (c),(f) Experimental spatial $S_{1}$ and $S_{2}$ Stokes parameters of the light transmitted through the cavity under tightly focused monochromatic antidiagonally polarized incident light. (d),(g) Corresponding patterns calculated from the analytical formula [Eq. (5)], and (e),(h) from Schrödinger equation simulation of Eq. (1).

Here $n_{x}$ and $n_{y}$ represent the refractive indices for linear polarizations in $x$ and $y$ directions, respectively, and $M, N$ denote the degenerate longitudinal cavity mode numbers of opposite parity, i.e., $M=N \pm 1$. The electric field of the modes $\Psi_{\vec{k}}^{ \pm}$in the vicinity of the mirrors is right- or lefthand circularly polarized with respect to the normal vector pointing outside the cavity plane. So a plus sign corresponds to an outgoing wave with $\vec{\sigma}_{+}=1 / \sqrt{2}[1, i]^{T}$ polarization vector, and the minus sign corresponds to an outgoing wave with the $\vec{\sigma}_{-}=1 / \sqrt{2}[1,-i]^{T}$ polarization vector on either side of the cavity. The planar symmetry of the cavity implies that incident electromagnetic waves of energy $\varepsilon$ and momentum $\vec{k}$ will excite modes $\varepsilon^{ \pm}(\vec{k})$. A linearly polarized incident plane wave with the polarization angle $\Theta=\frac{1}{2} \tan ^{-1}\left(S_{2} / S_{1}\right)$ with respect to the $x$ axis will excite either $e^{+i \Theta} \Psi^{+}$or $e^{-i \Theta} \Psi^{-}$waves inside the cavity, provided $\vec{k}$ belongs to the red or blue circle in Fig. 1(c). Therefore the 
transmitted light will be either right-hand or left-hand circularly polarized. If, however, the surface of the microcavity is illuminated with a focused coherent beam at normal incidence, then the entire isoenergy momentum spin circles are excited, and a specific polarization pattern determined by $\Theta$, as a manifestation of the optical PSH, will emerge. Let $F$ denote the distance between the focus of the incident beam and the illuminated surface. The electric field at this surface is a combination of plane waves (up to a common factor):

$$
\vec{E}_{\text {in }} \sim \int \frac{d^{2} k}{(2 \pi)^{2}} e^{i \vec{k} \cdot \vec{r}} e^{i R^{2} k^{2}}\left[\begin{array}{c}
\cos \Theta \\
\sin \Theta
\end{array}\right],
$$

where $R=\sqrt{F / 2 k_{0}}$ and $k_{0}$ is equal to the light wave number in the vacuum. Taking into account that each of those plane waves couples either to the field $e^{+i \Theta} \Psi^{+}$or $e^{-i \Theta} \Psi^{-}$ inside the cavity and performing the integral with respect to $\vec{k}$ one arrives at the final expression for the electric field of the transmitted wave at the opposite surface. Denoting $\quad \phi_{ \pm}(\vec{r}) \triangleq \mathcal{J}_{0}\left(\xi_{ \pm}\right)$, where $\mathcal{J}_{0}\left(\xi_{ \pm}\right)$is the Bessel function of zeroth order and $\xi_{ \pm}(\vec{r})=$ $\sqrt{2 m / \hbar^{2}\left(E+\left(2 m \alpha^{2} / \hbar^{2}\right)\right)}\left|\vec{r} \pm R^{2} \vec{Q}\right|$, we obtain

$\vec{E}_{\mathrm{out}} \sim e^{i[(\vec{Q} / 2) \cdot \vec{r}+\Theta]} \phi_{+}(\vec{r}) \vec{\sigma}_{+}+e^{-i[(\vec{Q} / 2) \cdot \vec{r}+\Theta]} \phi_{-}(\vec{r}) \vec{\sigma}_{-}$.

This expression exemplifies the so-called classical entanglement, while some authors find such terminology misleading [29], between the planar position and the polarization of the electric field [30]. Or alternatively, we have created an inseparable state between the valley $\pm \vec{Q} / 2$ and polarization $\vec{\sigma}_{ \pm}$degrees of freedom (d.o.f.) of the cavity photons. The spatial polarization pattern of the solution [Eq. (4)] can be represented by the components of the normalized Stokes vector,

$$
\begin{aligned}
& S_{1}=\frac{2 \phi_{+}(\vec{r}) \phi_{-}(\vec{r})}{\phi_{+}(\vec{r})^{2}+\phi_{-}(\vec{r})^{2}} \cos (\vec{Q} \cdot \vec{r}+2 \Theta), \\
& S_{2}=-\frac{2 \phi_{+}(\vec{r}) \phi_{-}(\vec{r})}{\phi_{+}(\vec{r})^{2}+\phi_{-}(\vec{r})^{2}} \sin (\vec{Q} \cdot \vec{r}+2 \Theta), \\
& S_{3}=\frac{\phi_{-}(\vec{r})^{2}-\phi_{+}(\vec{r})^{2}}{\phi_{+}(\vec{r})^{2}+\phi_{-}(\vec{r})^{2}} .
\end{aligned}
$$

The analytical results [Eq. (5)] are plotted in Figs. 2(d) and $2(\mathrm{~g})$ for $\Theta=-\pi / 4$, which agree with the experimental data obtained using antidiagonally polarized incident light. More strict analytical calculations taking into account the Gaussian profile of the focused beam do not change significantly the above conclusions. Additionally, we perform Schrödinger equation simulations $i \hbar \partial_{t} \Psi=\hat{H}(-i \nabla) \Psi+\vec{f}(\vec{r})$ driven by a normally incident antidiagonally polarized narrow Gaussian field $\vec{f}(\vec{r})=e^{-r^{2} /\left(2 w^{2}\right)}\left[e^{i \pi / 4},-e^{-i \pi / 4}\right]^{T}$ under open boundary conditions [28]. The results are shown in Figs. 2(e) and 2(h) in good agreement with the experiment and analytical theory. We note that the additional polarization circles appearing in Figs. 2(d) and 2(g) come from the focused incident light which picks up a spatially varying phase front, whereas in the Schrödinger simulations a driving field $\vec{f}(\vec{r})$ with flat phase front is used.

Our results open several new exciting perspectives in photonics. The so-called classically entangled d.o.f. between the cavity dispersion valleys and the photon spin offer a transparent and easy way to create inseparable photonic states [31]. Indeed, by controlling the polarization of the incident optical beam defined as $\vec{\sigma}_{\text {in }}=\beta_{+} \vec{\sigma}_{+}+\beta_{-} \vec{\sigma}_{-}$, where $\left|\beta_{+}\right|^{2}+\left|\beta_{-}\right|^{2}=1$, one can write Eq. (4) as a function of two control variables,

$$
\vec{E}_{\text {out }}\left(\beta_{ \pm}\right) \sim \beta_{+} e^{i \frac{\vec{Q}}{2} \cdot \vec{r}} \phi_{+}(\vec{r}) \vec{\sigma}_{+}+\beta_{-} e^{-i \frac{\vec{Q}}{2} \cdot \vec{r}} \phi_{-}(\vec{r}) \vec{\sigma}_{-} .
$$

It then becomes apparent that a family of inseparable states exists which satisfy $\left|\beta_{+}\right|=\left|\beta_{-}\right|$and $\beta_{+} / \beta_{-}=e^{2 i \Theta}$. Similar to a Michelson interferometer, $\Theta$ is an effective "pathdifference" variable which uniquely determines the location of the $S_{1,2}(\vec{r})$ interference minima and maxima in the PSH [see the Supplemental Material and Eq. (5)]. This reflects the fact that, for an inseparable state, any effects on one d.o.f. (e.g., spin) will have measurable outcome in the other d.o.f. (e.g., momentum valley) with exciting potential in optical metrology that benefits from parallelized d.o.f. measurements [32].

In the case of a tightly focused pump, as considered above, one has $\phi_{+}(\vec{r}) \simeq \phi_{-}(\vec{r})$, and the amount of nonseparability can be quantified as a global parameter $C=2\left|\beta_{+} \beta_{-}\right|$where $0 \leq C \leq 1$ [33]. When $C=1$ the system is maximally inseparable, whereas when $C=0$ the spin and valley degrees of freedom are completely separable. Analogous to the double slit experiment, where one can control the intensities of light passing through each slit affecting the interference pattern, setting $C<1$ describes different amounts of photons in the two circularly polarized components of light resulting in imbalanced occupation of the two valleys. In the more general case where $\phi_{+}(\vec{r}) \neq \phi_{-}(\vec{r})$ [causing the interference circles in the $S_{1,2}$ in Figs. 2(d) and 2(g)] the amount of inseparability is no longer a global quantity and depends on the spatial coordinate $\vec{r}$. In this case the $S_{3}(\vec{r})$ Stokes parameter becomes a useful measure of the amount of inseparability in space written as $C^{2}+S_{3}^{2}=1$, which is an analog of complementarity proposed by Eberly et al. [34].

Moreover, scrutinizing the $S_{3}(\vec{r})$ parameter we can demonstrate analogy of left- and right- hand circularly polarized light separation to an optical Stern-Gerlach experiment [35] using our system. The effective magnetic field of the equal Rasha-Dresselhaus SOC causes a 
spin-selective deflection of the cavity photons along the two opposite directions in the cavity plane defined by the valleys' location $\pm \vec{Q} / 2$ [Fig. 1(c)]. This deflection appears due to the different Bessel solutions for $\vec{\sigma}_{+}$and $\vec{\sigma}_{-}$which are shifted due to the anisotropy of the dispersion. This can be evidenced for the linearly polarized case $C=1$. For nonhomogeneous occupation of dispersion cavity valleys [Fig. 3(b)] we observe that $S_{3}(x, y)=-S_{3}(x,-y)$ as indicated theoretically in Fig. 3(c) and experimentally in Fig. 3(d). Such nonhomogeneous occupation of the valleys can be achieved by using a broad normal incident excitation beam which only excites a locality in reciprocal space around $k=0$. In the experiment presented in Fig. 3(d) we used linearly polarized (diagonal) light from a broadband halogen lamp transmitted through the sample by the same optical system. To filter energy of Rashba-Deresselhaus resonance we performed a tomography measurement by the motorized movement of a lens imaging light transmitted through the sample on the entrance slit of a monochromator equipped with a CCD camera. Our results therefore open a new method to design an optical Stern-Gerlach experiment in the classical optics regime. The notable difference between our setup and the actual Stern-Gerlach is that there is no constant force acting on the photon pseudospins (a)
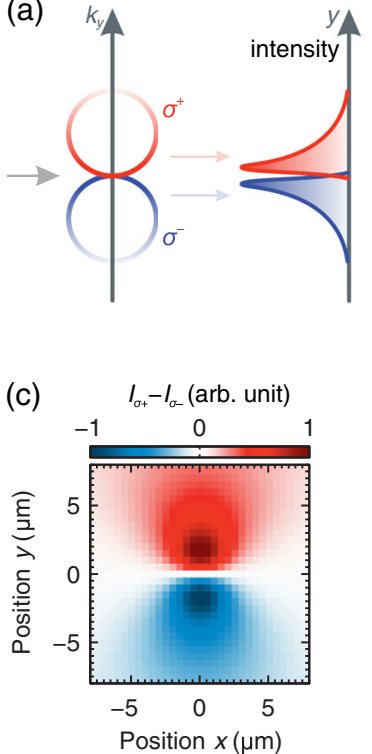

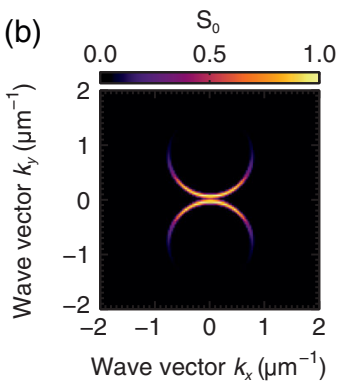

(d)

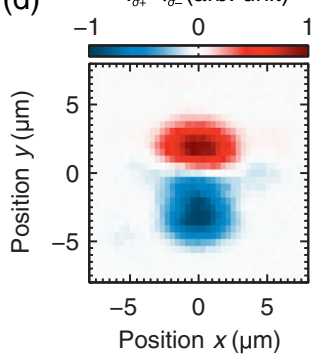

FIG. 3. Photonic Stern-Gerlach experiment. (a) Schematic of the reciprocal Stern-Gerlach experiment where the isoenergy circles of both valleys are partially populated resulting in separation of circular polarization along the $y$ direction. (b) Schrödinger simulations of the intensity of light in reciprocal space $\left(|\tilde{\Psi}(\vec{k})|^{2}\right)$ under linearly polarized wide Gaussian driving field $\vec{f}$. (c) Schrödinger simulations of the difference between transmitted light intensities in $\sigma^{+}$and $\sigma^{-}$polarizations. (d) Experimental difference between light intensities in $\sigma^{+}$and $\sigma^{-}$polarizations obtained from real space tomography under incoherent light transmission through the cavity using a wide Gaussian beam. but rather, they obtain constant group velocities in opposite directions in the cavity due to the effective magnetic force gradient [yellow arrows in Fig. 1(c)].

These results should not be confused with valley selective circular dichroism [36], as there are no absorption processes involved here, or Pancharatnam-Berry phase optical elements (which geometrically induce an optical spin Hall effect [37]) since our LC microcavity does not possess any cyclic parameters. Our results are also different from the spin Hall effect of light, also coming from geometric phases due to refractive index gradients [38], which are negligible compared with the birefringence induced by the LCs in our system. Moreover, since our system is achiral (i.e., the $x-z$ plane is a plane of symmetry) there is no circular birefringence, contrary to chiral materials like the Fresnel triprism [39]. A future path of investigation can then involve a quantum Stern-Gerlach experiment operating in the single photon regime. Finally, implementation of multiple spatially separated incident optical beams given by their central coordinates $\left\{\vec{r}_{1}, \vec{r}_{2}, \ldots\right\}$ introduces an additional external d.o.f. that defines the beams' location. Future photonic multi-d.o.f. experiments combining coordinate (i.e., incident beam central location), valley, and spin degrees of freedom can be engineered, producing more complex in plane polarization patterns as a result of interference.

In summary, we have investigated a liquid crystal-filled optical cavity with equal Rashba and Dresselhaus SOC contributions leading to $\mathrm{SU}(2)$ spin rotation symmetry [1]. An illustration of such symmetry is the emergence of the persistent spin helix, previously shown only in electronic systems [2,3]. This effect allows for fine control over the spatial polarization state of the in plane cavity photons on a micrometer scale. We have interpreted our observations in the framework of inseparable degrees of freedom played by the Rashba-Dresselhaus momentum valleys and the photon spin. This has allowed us to establish a reciprocal optical version of the famous Young's double slit experiment and the Stern-Gerlach experiment. Because of the compact design liquid crystal microcavity (LCMC) can be easily integrated with optoelectronics devices, giving the perspective of simulating complex spin systems, studying effects that are difficult to control in condensed matter systems, and developing photonic valleytronic devices in a relatively simple setting at room temperature.

This work was supported by the National Science Centre Grants No. 2019/35/B/ST3/04147 No. 2019/33/B/ST5/ 02658, No. 2018/31/N/ST3/03046, and No. 2017/27/B/ ST3/00271. This project has received funding from the European Union's Horizon 2020 research and innovation programme FET Open under grant agreement No. 964770 (TopoLight). H. S. and P. G. L. acknowledge the support of the UK's Engineering and Physical Sciences Research Council (Grant No. EP/M025330/1 on Hybrid 
Polaritonics), the support of the RFBR Projects No. 20-5212026 (jointly with DFG) and No. 20-02-00919, and the European Union's Horizon 2020 program, through a FET Open research and innovation action under the Grant Agreement No. 899141 (PoLLoC). H. S. acknowledges the Icelandic Research Fund, Grant No. 217631-051.

*These authors contributed equally to this work. Jacek.Szczytko@fuw.edu.pl

[1] B. A. Bernevig, J. Orenstein, and S.-C. Zhang, Exact SU(2) Symmetry and Persistent Spin Helix in a Spin-Orbit Coupled System, Phys. Rev. Lett. 97, 236601 (2006).

[2] J. D. Koralek, C. P. Weber, J. Orenstein, B. A. Bernevig, S.-C. Zhang, S. Mack, and D. D. Awschalom, Emergence of the persistent spin helix in semiconductor quantum wells, Nature (London) 458, 610 (2009).

[3] M. P. Walser, C. Reichl, W. Wegscheider, and G. Salis, Direct mapping of the formation of a persistent spin helix, Nat. Phys. 8, 757 (2012).

[4] M. Kohda, V. Lechner, Y. Kunihashi, T. Dollinger, P. Olbrich, C. Schönhuber, I. Caspers, V. V. Bel'kov, L. E. Golub, D. Weiss, K. Richter, J. Nitta, and S. D. Ganichev, Gate-controlled persistent spin helix state in (In,Ga)As quantum wells, Phys. Rev. B 86, 081306(R) (2012).

[5] J. Ishihara, Y. Ohno, and H. Ohno, Direct imaging of gatecontrolled persistent spin helix state in a modulation-doped GaAs/AlGaAs quantum well, Appl. Phys. Express 7, 013001 (2014).

[6] A. Sasaki, S. Nonaka, Y. Kunihashi, M. Kohda, T. Bauernfeind, T. Dollinger, K. Richter, and J. Nitta, Direct determination of spin-orbit interaction coefficients and realization of the persistent spin helix symmetry, Nat. Nanotechnol. 9, 703 (2014).

[7] M. Kohda and G. Salis, Physics and application of persistent spin helix state in semiconductor heterostructures, Semicond. Sci. Technol. 32, 073002 (2017).

[8] J. Schliemann, Colloquium: Persistent spin textures in semiconductor nanostructures, Rev. Mod. Phys. 89, 011001 (2017).

[9] F. Passmann, S. Anghel, T. Tischler, A. V. Poshakinskiy, S. A. Tarasenko, G. Karczewski, T. Wojtowicz, A. D. Bristow, and M. Betz, Persistent spin helix manipulation by optical doping of a CdTe quantum well, Phys. Rev. B 97, 201413(R) (2018).

[10] K. Y. Bliokh, F. J. Rodríguez-Fortuño, F. Nori, and A. V. Zayats, Spin-orbit interactions of light, Nat. Photonics 9 , 796 (2015).

[11] T. Ozawa, H. M. Price, A. Amo, N. Goldman, M. Hafezi, L. Lu, M. C. Rechtsman, D. Schuster, J. Simon, O. Zilberberg, and I. Carusotto, Topological photonics, Rev. Mod. Phys. 91, 015006 (2019).

[12] C. Leyder, M. Romanelli, J. P. Karr, E. Giacobino, T. C. H. Liew, M. M. Glazov, A. V. Kavokin, G. Malpuech, and A. Bramati, Observation of the optical spin Hall effect, Nat. Phys. 3, 628 (2007).

[13] M. Król, H. Sigurdsson, K. Rechcińska, P. Oliwa, K. Tyszka, W. Bardyszewski, A. Opala, M. Matuszewski, P. Morawiak, R. Mazur, W. Piecek, P. Kula, P. G. Lagoudakis,
B. Piętka, and J. Szczytko, Observation of second-order meron polarization textures in optical microcavities, Optica 8, 255 (2021).

[14] N. Shitrit, I. Yulevich, E. Maguid, D. Ozeri, D. Veksler, V. Kleiner, and E. Hasman, Spin-optical metamaterial route to spin-controlled photonics, Science 340, 724 (2013).

[15] S.-H. Gong, F. Alpeggiani, B. Sciacca, E. C. Garnett, and L. Kuipers, Nanoscale chiral valley-photon interface through optical spin-orbit coupling, Science 359, 443 (2018).

[16] N. Lundt, Ł. Dusanowski, E. Sedov, P. Stepanov, M. M. Glazov, S. Klembt, M. Klaas, J. Beierlein, Y. Qin, S. Tongay, M. Richard, A. V. Kavokin, S. Höfling, and C. Schneider, Optical valley Hall effect for highly valleycoherent exciton-polaritons in an atomically thin semiconductor, Nat. Nanotechnol. 14, 770 (2019).

[17] C. E. Whittaker, T. Dowling, A. V. Nalitov, A. V. Yulin, B. Royall, E. Clarke, M. S. Skolnick, I. A. Shelykh, and D. N. Krizhanovskii, Optical analogue of Dresselhaus spin-orbit interaction in photonic graphene, Nat. Photonics 15, 193 (2021).

[18] K. Rechcińska, M. Król, R. Mazur, P. Morawiak, R. Mirek, K. Łempicka, W. Bardyszewski, M. Matuszewski, P. Kula, W. Piecek, P. G. Lagoudakis, B. Piętka, and J. Szczytko, Engineering spin-orbit synthetic Hamiltonians in liquidcrystal optical cavities, Science 366, 727 (2019).

[19] Y.-J. Chen, J. D. Cain, T. K. Stanev, V. P. Dravid, and N. P. Stern, Valley-polarized exciton-polaritons in a monolayer semiconductor, Nat. Photonics 11, 431 (2017).

[20] Z. Sun, J. Gu, A. Ghazaryan, Z. Shotan, C. R. Considine, M. Dollar, B. Chakraborty, X. Liu, P. Ghaemi, S. Kéna-Cohen, and V.M. Menon, Optical control of room-temperature valley polaritons, Nat. Photonics 11, 491 (2017).

[21] S. Dufferwiel, T. P. Lyons, D. D. Solnyshkov, A. A. P. Trichet, F. Withers, S. Schwarz, G. Malpuech, J. M. Smith, K. S. Novoselov, M. S. Skolnick, D. N. Krizhanovskii, and A. I. Tartakovskii, Valley-addressable polaritons in atomically thin semiconductors, Nat. Photonics 11, 497 (2017).

[22] R. Dąbrowski, P. Kula, and J. Herman, High birefringence liquid crystals, Crystals 3, 443 (2013).

[23] E. Miszczyk, R. Mazur, P. Morawiak, M. Mrukiewicz, W. Piecek, Z. Raszewski, P. Kula, K. Kowiorski, J. Kędzierski, and J. Zieliński, Refractive index matched liquid crystal cell for laser metrology application, Liq. Cryst. 45, 1690 (2018).

[24] K. Lekenta, M. Król, R. Mirek, K. Lempicka, D. Stephan, R. Mazur, P. Morawiak, P. Kula, W. Piecek, P. G. Lagoudakis, B. Pietka, and J. Szczytko, Tunable optical spin Hall effect in a liquid crystal microcavity, Light Sci. Appl. 7, 74 (2018).

[25] J. Ren, Q. Liao, F. Li, Y. Li, O. Bleu, G. Malpuech, J. Yao, H. Fu, and D. Solnyshkov, Nontrivial band geometry in an optically active system, Nat. Commun. 12, 689 (2021).

[26] D. W. Berreman, Optics in stratified and anisotropic media: $4 \times 4$-matrix formulation, J. Opt. Soc. Am. 62, 502 (1972).

[27] M. Schubert, Polarization-dependent optical parameters of arbitrarily anisotropic homogeneous layered systems, Phys. Rev. B 53, 4265 (1996).

[28] See Supplemental Material at http://link.aps.org/ supplemental/10.1103/PhysRevLett.127.190401 for details.

[29] E. Karimi and R. W. Boyd, Classical entanglement?, Science 350, 1172 (2015). 
[30] A. Forbes, A. Aiello, and B. Ndagano, Classically entangled light, in Progress in Optics, edited by T. D. Visser (Elsevier, Amsterdam, 2019), Vol. 64, pp. 99-153.

[31] K. H. Kagalwala, G. Di Giuseppe, A. F. Abouraddy, and B. E. A. Saleh, Bell's measure in classical optical coherence, Nat. Photonics 7, 72 (2013).

[32] F. Töppel, A. Aiello, C. Marquardt, E. Giacobino, and G. Leuchs, Classical entanglement in polarization metrology, New J. Phys. 16, 073019 (2014).

[33] N. Korolkova and G. Leuchs, Quantum correlations in separable multi-mode states and in classically entangled light, Rep. Prog. Phys. 82, 056001 (2019).

[34] J. H. Eberly, X.-F. Qian, A. A. Qasimi, H. Ali, M. A. Alonso, R. Gutiérrez-Cuevas, B. J. Little, J. C. Howell, T. Malhotra, and A. N. Vamivakas, Quantum and classical optics-emerging links, Phys. Scr. 91, 063003 (2016).
[35] L. Karpa and M. Weitz, A Stern-Gerlach experiment for slow light, Nat. Phys. 2, 332 (2006).

[36] T. Cao, G. Wang, W. Han, H. Ye, C. Zhu, J. Shi, Q. Niu, P. Tan, E. Wang, B. Liu, and J. Feng, Valley-selective circular dichroism of monolayer molybdenum disulphide, Nat. Commun. 3, 887 (2012).

[37] N. Shitrit, I. Bretner, Y. Gorodetski, V. Kleiner, and E. Hasman, Optical spin Hall effects in plasmonic chains, Nano Lett. 11, 2038 (2011).

[38] O. Hosten and P. Kwiat, Observation of the spin Hall effect of light via weak measurements, Science 319, 787 (2008).

[39] O. Arteaga, E. Garcia-Caurel, and R. Ossikovski, SternGerlach experiment with light: Separating photons by spin with the method of A. Fresnel, Opt. Express 27, 4758 (2019). 